\title{
Molecular epidemiology of carbapenem resistant Enterobacteriaceae in Sri Lanka: First report of KPC- producing Klebsiella pneumoniae
}

Wirittamulla Gamage Maheshika Kumudunie

University of Kelaniya/Faculty of Medicine

Wijesooriya Rathnayaka Pathirennehalage Lakmini Inoka Wijesooriya

University of Kelaniya/Faculty of Medicine

Kalubowilage Dhananja Namalie

Colombo North Teaching Hospital

Narapity Pathirannehalage Sunil-Chandra

University of Kelaniya/Faculty of Medicine

Yasanandana Supunsiri Wijayasinghe ( $\nabla$ supunw@kln.ac.lk)

University of Kelaniya/Faculty of Medicine https://orcid.org/0000-0002-1269-6397

Research article

Keywords: Enterobacteriaceae, multidrug-resistant, ESBL producing, carbapenem-resistant, carbapenemase producing, Sri Lanka

Posted Date: April 22nd, 2020

DOI: https://doi.org/10.21203/rs.2.9753/v2

License: (c) (1) This work is licensed under a Creative Commons Attribution 4.0 International License.

Read Full License 
The authors have withdrawn this preprint from Research Square 